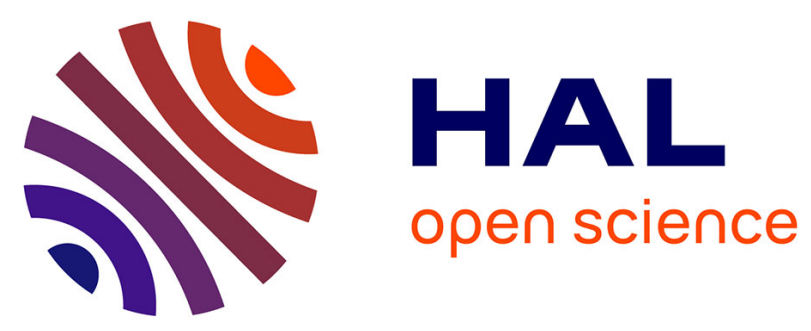

\title{
Using Wearable Monitors to Assess Daily Walking Limitations Induced by Ischemic Pain in PAD
}

Ségolène Chaudru, Pierre Jehannin, Pierre-Yves de Müllenheim, Hélène Klein, Vincent Jaquinandi, Guillaume Mahé, Alexis Le Faucheur

\section{- To cite this version:}

Ségolène Chaudru, Pierre Jehannin, Pierre-Yves de Müllenheim, Hélène Klein, Vincent Jaquinandi, et al.. Using Wearable Monitors to Assess Daily Walking Limitations Induced by Ischemic Pain in PAD. Scandinavian Journal of Medicine and Science in Sports, 2019, 29 (11), pp.1813-1826. 10.1111/sms.13511 . hal-02179151

HAL Id: hal-02179151

https://hal-univ-rennes1.archives-ouvertes.fr/hal-02179151

Submitted on 14 Oct 2019

HAL is a multi-disciplinary open access archive for the deposit and dissemination of scientific research documents, whether they are published or not. The documents may come from teaching and research institutions in France or abroad, or from public or private research centers.
L'archive ouverte pluridisciplinaire HAL, est destinée au dépôt et à la diffusion de documents scientifiques de niveau recherche, publiés ou non, émanant des établissements d'enseignement et de recherche français ou étrangers, des laboratoires publics ou privés. 
DR GUILLAUME MAHÉ (Orcid ID : 0000-0003-1318-4745)

MR ALEXIS LE FAUCHEUR (Orcid ID : 0000-0002-3688-6574)

Article type : Original Article

\section{Using Wearable Monitors to Assess Daily Walking Limitations Induced by Ischemic Pain in PAD}

Running title: Daily life walking pain in people with PAD

\section{Authors}

Ségolène Chaudru, $\mathrm{MSc}^{1,2}$; Pierre Jehannin $\mathrm{MSc}^{1,2}$; Pierre-Yves de Müllenheim, $\mathrm{PhD}^{3}$;

Hélène Klein, $\mathrm{BSc}^{4}$; Vincent Jaquinandi, $\mathrm{MD}, \mathrm{PhD}^{1,2,5}$; Guillaume Mahé, $\mathrm{MD}, \mathrm{PhD}^{1,2,5}$; and Alexis Le Faucheur, $\mathrm{PhD}^{1,3,4}$

Ségolène Chaudru and Pierre Jehannin contributed equally to this article

\section{Affiliations}

${ }^{1}$ Clinical Investigation Center, INSERM CIC 1414, F-35033 Rennes, France

${ }^{2}$ Univ Rennes 1, Rennes, France

${ }^{3}$ Univ Rennes, M2S - EA 7470, F-35000 Rennes, France

${ }^{4}$ Department of Sport sciences and physical education. Ecole normale supérieure de Rennes

Campus de Ker Lann. Bruz, F-35170, France.

${ }^{5}$ Unité de médecine vasculaire, CHU, F-35033 Rennes, France. 


\section{Corresponding author: Alexis LE FAUCHEUR}

Department of Sport sciences and physical education. Ecole normale supérieure de Rennes, Campus de Ker Lann. Bruz, F-35170, France.

Tel.: +33 (0)2 9905 9419. Fax: +33 (0)2 9905 9329. E-mail: alexis.lefaucheur@ens-rennes.fr

\section{ABSTRACT}

PURPOSE: To develop, assess the feasibility of, and determine the clinical validity of an event-based analysis method using wearable monitors to quantify walking pain manifestations (WPMs) and stops induced by walking pain (SIWPs) during daily life walking in people with peripheral artery disease (PAD).

METHODS: The following two walking conditions were studied: a standardized outdoor walking session (OWS) and a 7-day free-living measurement (FLM) period. The PAD participants $(n=23)$ wore an accelerometer and a watch. They were asked to press the event marker button on the watch to indicate events related to WPMs and SIWPs. To assess the clinical validity of the method, the computed pain-free walking time (PFWT) and maximal walking time (MWT) were compared to the PFWT and MWT assessed using standard treadmill walking protocols, respectively.

RESULTS: Following OWSs, the PFWT ${ }^{[\mathrm{OWS}]}$ and MWT ${ }^{[\mathrm{OWS}]}$ were significantly correlated with the PFWT ${ }^{[\text {Strandness }]}(\mathrm{r}=0.955, \mathrm{P}<0.001)$ and $\mathrm{MWT}^{[\text {Strandness }]}(\mathrm{r}=0.821, \mathrm{P}<0.001)$, respectively. During the FLM, PAD participants experienced only 2 WPMs/day and 1 SIWP/day, although severely limited on the treadmill and during the OWS. The average WPMs/day were moderately correlated with the $\mathrm{PFWT}^{[\text {Strandness }]}(\mathrm{r}=-0.54, \mathrm{P}=0.016)$. The $\mathrm{PFWT}^{[\mathrm{FLM}]}$ was on average 12 times longer than the PFWT ${ }^{[\text {Strandness }]}$. Interestingly, the 
intensity of the walking bouts as assessed by the accelerometer counts during the FLM was significantly lower than that during the OWS $(45 \pm 15$ vs. $66 \pm 21$ counts/s, $\mathrm{P}<0.001)$.

CONCLUSION: This new method provides perspectives for studies investigating the experience of living with PAD and the assessment of daily life walking capacity for both diagnostic and therapeutic purposes.

KEY WORDS: physical activity; walking; intermittent claudication; accelerometry; event history analysis

\section{Clinical Trail Registration: https://clinicaltrials.gov/ct2/show/NCT02041169}

\section{INTRODUCTION}

Lower extremity peripheral artery disease (PAD) is a chronic atherosclerotic occlusive disease that causes insufficient blood flow to the lower extremities, which can manifest as ischemic walking pain in the lower limbs and impair walking in people with $\mathrm{PAD}^{1}$. For both diagnostic and therapeutic purposes, assessing lower limb pain manifestation and the associated walking impairment is an important aspect of PAD management ${ }^{2}$. The "gold standard" method for such an assessment is the measurement of the pain-free walking time (PFWT) or distance and the maximal walking time (MWT) or distance during a treadmill walking test ${ }^{2}$. The MWT has been reported to be more reliably estimated than the PFWT $^{3}$. However, the joint estimation of PFWT and MWT during walking crucial for fully assessing the pain experienced by people with PAD particularly because different treatment options can differentially impact the pain trajectory during walking ${ }^{4}$, leading to independent changes in the PFWT and $\mathrm{MWT}^{5}$. 
Although the treadmill assessment of the PFWT and MWT is well standardized and has been extensively studied ${ }^{3}$, it remains protocol dependent, technically demanding, time-consuming, and limited to vascular laboratories. Furthermore, the maximal treadmill walking distance can exhibit substantial variation relative to the "true" outdoor walking capacity while walking at a usual pace on a flat surface ${ }^{6-8}$ and does not reflect the patient's perceived disability ${ }^{3,9,10}$. This issue is a serious concern for clinical decision-making and research purposes because the consensus opinion supports that the optimal functional test should be correlated with patientreported ambulatory function in daily life $\mathrm{e}^{11-14}$.

An accurate ambulatory measurement of the outdoor walking capacity has been proposed using Global Positioning System (GPS) receivers ${ }^{6,7,15,16}$. However, as currently used, the onset of walking pain cannot be retrieved from an analysis of a GPS speed signal, which precludes the estimation of the PFWT and a full assessment of the pain experienced during "natural" outdoor walking. Furthermore, the GPS assessment of the daily walking capacity is limited in time (a 45- to 60-minute outdoor walking session (OWS)), space (outdoors), feasibility (may depend on the weather conditions), and clinical interpretation (stops having to be induced by lower limb pain only), which may preclude an extended analysis of the walking capacity under complete free-living conditions ${ }^{7}$. Therefore, using seven-day continuous ambulatory monitoring with an activPAL ${ }^{\mathrm{TM}}$ monitor, Clarke et al. developed a method for performing an event-based analysis to quantify the fragmented nature of walking bouts in people with PAD with intermittent claudication ${ }^{17}$. However, this method did not enable clinicians to determine whether the stops between bouts of walking were due to exertional pain induced by ischemia. Thus, a method for assessing walking pain manifestations (WPMs) is required to fully assess and understand the pattern of lower limb pain manifestation and the related functional impairment during daily life walking ${ }^{18}$. 
The aims of this study were as follows: (i) the assessment of the feasibility of tracking and quantifying WPMs and stop(s) induced by walking pain (SIWPs) using wearable monitors during an OWS in PAD participants; (ii) development of an event-based analysis method using wearable monitors to quantify WPMs and SIWPs during daily life in PAD participants; and (iii) determination of the clinical validity of the computed PFWT and MWT derived from the recording of WPMs and SIWPs, respectively.

\section{MATERIALS AND METHODS}

\section{Study overview}

This cross-sectional study was a part of the "CLASH" project (NCT02041169), which was approved by the local institutional ethics committee of Rennes (CPP OUEST V, Rennes, France). People with PAD were recruited from our Vascular Medicine Unit (University Hospital, Rennes, France) during their medical appointment, which included a treadmill test (eligibility visit). The subjects who met the inclusion criteria (Table 1) were invited to participate. Then, within a 1-month period, the included PAD participants underwent the following: a laboratory assessment of their walking capacity, a 45-60 min OWS, and a 7-day free-living measurement (FLM).

The aim of the OWS was first to determine the ability of the PAD participants to record events related to ischemic pain while walking (i.e., WPM and SIWP) using a Micro Motionlogger® Watch worn at the wrist level. Second, we aimed to determine the PFWT and the MWT based on the WPM and SIWP events, respectively, to analyze their associations with the treadmill measures and assess the clinical validity of these assessments. The aim of the 7-day FLM was to implement an event-based analysis by combining the data 
from the watch and accelerometer to determine the free-living walking limitations (PFWT and MWT) and their association with the treadmill measures.

\section{Experimental procedure}

Laboratory assessment of the walking capacity. During the first visit (inclusion visit), the participants' sex, weight, height, cardiovascular risk factors, comorbid conditions, claudication history and list of current medications were obtained through the medical histories and medical reports. Self-reported ambulatory walking impairment was assessed using the WIQ, and the quality of life was evaluated using the SF-36 questionnaire. The lower limb symptoms experienced by the PAD participants were assessed and classified using the San Diego claudication questionnaire ${ }^{19}$ and the categories of leg symptoms modified by McDermott et al. ${ }^{20}$ The resting ankle-brachial index was measured using a handheld Doppler probe according to the recommendations of the American Heart Association $^{21}$. Then, the PAD participants performed a Gardner-Skinner treadmill protocol $(3.2 \mathrm{~km} / \mathrm{h}, 0 \%$ grade with $2 \%$ increase every 2 minutes) until reaching maximal claudication pain. On another day, the PAD participants also performed a Strandness treadmill protocol $(3.2 \mathrm{~km} / \mathrm{h}, 10 \%$ grade) until reaching maximal claudication pain. During both treadmill tests, the PFWT and the MWT were recorded.

Outdoor walking session. The PAD participants performed an OWS on a dedicated day that consisted of walking at their usual walking speed for 45 to $60 \mathrm{~min}$, including eventual bouts of stopping (recovery) due to lower-limb pain. The participants were asked to stop walking only due to lower-limb pain and were given no recommendations regarding the minimal or maximal duration of the stops. The OWSs occurred on a designated athletic track ( $0 \%$ grade) that was free of buildings and compact trees. Rainy days were avoided. 
The PAD participants were equipped with (i) a Micro Motionlogger® Watch (Ambulatory Monitoring, Inc., Ardsley, NY; firmware Action-W version 2); (ii) a DG100 GPS receiver (GlobalSat, Taipei, Taiwan); and (iii) a wGT3X+ accelerometer $\left(\right.$ ActiGraph $^{\mathrm{TM}}, \mathrm{LLC}$, Pensacola, FL; firmware version: 2.2.1 to 2.4.0). The watch was used to record the WPMs and SIWPs. This device is a small $(3.6 \times 3.6 \times 1.2 \mathrm{~cm})$ and light $(30 \mathrm{~g})$ digital wrist watch-sized microprocessor that senses motion and enables the user to generate an event marker in the recording data file. The device was initialized at a 5-s recording epoch. The DG100 GPS receiver (dimensions $80 \times 55 \times 18 \mathrm{~mm}$, weight $\sim 200 \mathrm{~g}$ ) was used to assess the walking capacity of the PAD participants during the OWS as previously described ${ }^{15}$. The DG100 includes the European Geostationary Navigation Overlay Service function, which was activated during the measurements. The DG100 was set at a 1-Hz recording rate using a personal computer and the manufacturer's software (Data Logger Utility, version 1.1). Then, the DG100 unit was placed into a plastic pocket with an external antenna (AT-65 GPS Active Antenna; GlobalSat) fixed to the top of the pocket shoulder strap. The wGT3X+ is a small $(4.6 \times 3.3 \times 1.5 \mathrm{~cm})$ and light $(19 \mathrm{~g})$ tri-axial accelerometer that was used to quantify the intensity of the walking bouts for the subsequent comparison with the 7-day FLM. This device was initialized at a 30-Hz recording rate using Actilife 6 software (ActiGraphTM, LLC, Pensacola, FL; version 6.5.3), placed in a nylon pouch with the Velcro closures supplied by the manufacturer and placed at the right hip (on the iliac crest). The monitors were synchronized using the same computer clock (Universal Time Coordinated). During the OWS, the participants were asked to use the watch and push a specific marker button to record (i) pain manifestations in the lower limbs while walking (WPMs) and (ii) stops due to lower limb pain (SIWPs). To record a WPM, the participants were asked to press the marker button of the watch once. To record an SIWP, the participants were asked to consecutively press the marker button twice. 
The walking session was supervised by two investigators who walked approximately 10 meters behind the participant. During the walking session, using a chronometer, the investigators reported the time when the patient pressed the marker button of the watch to indicate a WPM or SIWP.

7-day free-living measurement. The participants were asked to wear a wGT3X+ and the watch for seven consecutive days. The devices were initialized and worn as described during the OWS (see above). Notably, for the watch, the 5-s recording rate was the highest recording rate available for recording data for at least seven full days. The monitors were synchronized using the same computer clock (Universal Time Coordinated). The participants were carefully instructed to wear the wearable monitors throughout the day for seven consecutive days and remove the monitors during water-based activities (e.g., showering and swimming) and at bedtime (the watch could be worn during the night at the convenience of the patient). Throughout the entire recording period, the participants were asked to push the event marker button of the watch to indicate a WPM (1 push) and an SIWP ( $\geq 2$ pushes). The participants were asked to perform their activities as usual. At the end of each day, the participants reported the main indoor and/or outdoor activities carried out throughout the day in a specific notebook with a timestamp. A booklet containing all instructions regarding the use of the monitors and completion of the notebook was also delivered to each patient. During the entire recording period, the participants received a short message each morning to remind them to wear the monitors. At the end of the 7-day monitoring period, a visit was scheduled, and the participants returned the monitors. An investigator also asked the participants questions regarding any discomfort caused by the monitors and protocol compliance. 


\section{Data analysis}

Questionnaires. The responses to the WIQ and SF-36 questionnaires were recorded using a spreadsheet and scored following the recommended procedures ${ }^{22-25}$. Each component of the WIQ was scored to obtain the WIQ ${ }^{[\text {Distance }]}$ WIQ $^{[\text {Speed] }}$ and WIQ ${ }^{[\text {Stairs] }}$ subscale scores. A WIQ total score $\left(\mathrm{WIQ}{ }^{[\text {Total] }}\right.$ ) was also computed by averaging the three subscale scores. The WIQ scores ranged between 0 and $100 \%$, with $100 \%$ indicating no impairment. Following the scoring of the SF-36, 8 scale scores for 8 items were computed; each item reflects different domains of the quality of life, and each domain is scored on a scale ranging from 0 to 100 , with 100 representing the highest possible score (best possible health status).

Outdoor walking session. For each walking session, the mean values of the weather parameters (temperature $\left(\mathrm{C}^{\circ}\right)$, relative humidity $(\mathrm{RH}$ in $\%)$, wind speed $(\mathrm{km} / \mathrm{h})$, and barometric pressure $(\mathrm{hPa})$ ) were recorded using the information provided by French weather stations. The event markers recorded by the watch were also downloaded to a computer using Ambulatory Monitoring software (Action-W version 2). These markers were available in a specific column of the data file and processed using Excel® 2016 (Microsoft, Redmond, WA). For each watch data file, we determined whether an event marker was inserted into the data file and whether it was consistent with the event observed by the investigators (i.e., a WPM or SIWP). We also identified the time stamp corresponding to the WPM or SIWP recorded in the data file and the number of times the button was pressed to record the event. We also determined the time difference between the watch-recorded and observed WPMs and SIWPs. Finally, for each WPM and SIWP identified from the watch, we computed the PFWT and the MWT of the OWS (PFWT ${ }^{[\mathrm{OWS}]}$ and $\mathrm{MWT}^{[\mathrm{OWS}]}$, respectively). For each patient, we computed both the average (from all walking bouts) and the higher (longer walking bout) $\mathrm{PFWT}^{[\mathrm{OWS}]}$ and $\mathrm{MWT}^{[\mathrm{OWS}]}$. 
The raw acceleration data recorded by the wGT3X+ were downloaded to a computer. Then, the raw data were accumulated into 1-s epoch data (i.e., into counts/s) using the Low Frequency Extension (LFE) filter available in the Actilife 6 software (version 6.5.3) to compute the counts/s on the vector magnitude (VM). Then, for each walking and stopping bout identified from the GPS analysis, the mean \pm standard deviation of the VM was calculated. The GPS data were downloaded to a personal computer using the manufacturer's software (DataLogger Utility, version 1.1) and processed according to a previously published procedure $^{7,26}$. The GPS parameters recorded for each patient included the total distance walked, the entire duration of the outdoor session, the number of walking bouts, the mean walking speed calculated over each walking bout, the various walking times between two SIWPs and over each walking bout, the higher MWT between two SIWPs per patient during the entire walking session, and the duration of the stop preceding each walking bout.

7-day free-living measurement. Both the $\mathrm{WGT} 3 \mathrm{X}+$ and the watch data were downloaded as described for the OWS. The data files were exported to spreadsheets for further analysis. Identifying valid days. The wGT3X+ and watch data were screened for valid wear days. A valid wear day consisted of at least 10 hours of wear time. From the watch recording, the wear time periods were identified using the "Life measures signal" function as suggested by the manufacturer. These life measurements sense body vibrations from essential tremors and the ballistic heart signal. The absence of this signal is an indication that the watch is no longer on the body. From the wGT3X+ recordings, the nonwear time periods were identified using the method described by Choi et $\mathrm{al}^{27}$. Briefly, the nonwear time was defined as 90 consecutive minutes of zero counts ( $\mathrm{V}$-axis counts), with an allowance of 2-minute intervals of nonzero counts with an up/downstream 30-minute consecutive zero count window. 
Matching the data. The data from each monitor were processed using a custom MATLAB ${ }^{\circledR}$ code to generate a single Excel $^{\circledR}$ spreadsheet per participant that contains the data recorded by each wearable monitor, and the data were synchronized with each other and a one-second time stamp.

Labeling event markers from the Micro Motionlogger ${ }^{\mathrm{TM}}$ watch. We developed an event-based analysis method to label each event marker recorded by the watch, i.e., WPMs and SIWPs. The labeling methodology was applied to each event marker that appeared during a day considered valid for both the watch and wGT3X+ data. The methodology is presented in Figure 1. As shown in Figure 1 (left panel), the methodology first relied on discriminating the walking bouts from the non-walking bouts using the VM count data recorded by the wGT3X+. This approach consisted of using a counts/s threshold as previously proposed to identify sedentary behavior in adults in free-living environments ${ }^{28}$. A bout was considered a walking bout if the count/s values were above the predefined count threshold for at least $15 \mathrm{~s}$. In contrast, a bout was considered a stopping bout if the count/s values were below the predefined count threshold for at least $15 \mathrm{~s}$. The rationale for using a minimum duration of 15 s to define the walking and non-walking bouts was that walking-induced ischemia was unlikely to appear (during walking) or disappear (during stopping) within such a short period $^{15,16}$. Then, each event was assigned to the following three distinct categories: (i) WPM; (ii) SIWP; (iii) or "inconsistent". For both WPMs and SIWPs, we removed duplicate events related to ischemic events (multiple presses related to the same WPM or SIWP event performed by the participants to ensure that the events were recorded). Otherwise, all other events were labeled "inconsistent”. Finally, for each WPM and SIWP identified from the watch, we computed the PFWT and the MWT of the FLM (PFWT ${ }^{[\mathrm{FLM}]}$ and MWT ${ }^{[\mathrm{FLM}]}$, respectively). For each patient, we computed both the average (from all walking bouts) and the highest (longer walking bouts) $\mathrm{PFWT}^{[\mathrm{FLM}]}$ and $\mathrm{MWT}^{[\mathrm{FLM}]}$. 
Coding diaries. The notebook information was coded using the Compendium of Physical Activities $^{29}$. Finally, each event was matched using the encoding notebook and then analyzed. A typical example of event identification and qualification is illustrated in Figure 1 (right panel, A \& B).

Physical activity level. The physical activity level was assessed as the time spent in the different physical activity categories and was determined using the $\mathrm{V}$-axis counts recorded by the wGT3X+ (sedentary: <100 counts/min; light: between 100 and 759 counts/min; moderate: between 760 and 5724 counts/min; and vigorous: $>5724$ counts/min) ${ }^{30}$. The time spent in the different physical activity categories was also expressed as a percentage of the wear time (\%/day). We also calculated the number of bouts $\geq 10$ minutes with a $\mathrm{V}$-axis level $\geq 760$ counts/minute. Because WPMs are expected to occur during the continuous walking period, no drop time was allowed. The metrics were computed with Actilife ${ }^{\circ}$ software (ActiGraphTM, LLC, Pensacola, FL; version 6.5.3).

\section{Statistical analysis}

The normal distribution of the data of each variable of interest was assessed using the Shapiro-Wilk test. Accordingly, the data of the continuous variables are reported as the mean \pm standard deviation or medians with the interquartile range ( $\mathrm{IQR}=25^{\text {th }}-75^{\text {th }}$ percentiles), while the data of the categorical variables are expressed as percentages. The pairwise correlations between variables of interest were investigated by calculating the Pearson or Spearman correlation coefficients according to the distribution of the data. In addition, pairwise comparisons were performed using either the paired samples t-test or Wilcoxon signed-rank test according to the distribution of the data. Multiple comparisons of the PFWT or MWT between each condition (Strandness, Gardner, OWS, and FLM) were performed using the Friedman test (non-normal data). For $\mathrm{P}<0.05$, following the Friedman test, a 
pairwise comparison was performed between each pair of variables using the Wilcoxon Signed-Rank test with a Bonferroni correction of the P-values. All statistical analyses were performed using SPSS software (version 23.0.0.2). The level of significance was set at $\mathrm{P}<0.05$.

\section{RESULTS}

\section{Characteristics of the included PAD participants}

In total, 23 participants were included in the present study. The participants' characteristics are presented in Table 2. The mean age of the included PAD participants was $60 \pm 10$ years, and most (87\%) participants were male subjects. The median ABI [IQR] was 0.61 [0.53;0.75], indicating a mild to moderate PAD severity. Two PAD participants had to stop their participation before the end of the study, resulting in two missing Strandness walking tests. Due to the uncontrolled treatment of hypertension, one of the two participants experienced a severe episode of orthostatic hypotension during the recovery from the Strandness treadmill walking test. The other patient underwent vascular intervention. However, the results of the Gardner walking test, the OWS and the FLM were available for these two participants. During the treadmill tests, all PAD participants experienced lower limbs pain forcing them to stop walking and that was relieved or lessened within 10 minutes of rest. Following the Gardner-Skinner treadmill test, the PFWT and MWT were $131 \mathrm{~s}$ [89;224] and 289 s [203;420], respectively. Following the Strandness treadmill test, the PFWT and MWT were $79 \mathrm{~s}$ [56;105] and $178 \mathrm{~s}$ [116;213], respectively. 


\section{Outdoor walking session}

Table 3 presents an overview of the data obtained from the 23 participants during the OWS. All PAD participants reported walking pain during the OWS, and the median [IQR] number of stops induced by pain was 6 [4-10].

Feasibility of event recording using the watch. Observation-based events were not reported by the investigators in one subject, and in another subject, the watch was not available on the day of the OWS. In three other subjects, in total, five events were not reported by the investigators. Overall, the validity of the event recording using the watch could be studied in 21 subjects and over 170 walking bouts.

As shown in Table 3, in total, 126 WPMs were observed by the investigators. The number of WPMs recorded by the watch was 115 (91\%). At the patient level, the missing WPM events only involved three participants, and more than $70 \%$ of the missing events were related to one patient. In total, 170 SIWPs were observed by the investigators. The number of SIWPs was higher than the number of WPMs because some PAD participants resumed their walk before the pain in their lower limb(s) had fully disappeared ( $n=7$ participants). Over the 170 SIWPs, $153(90 \%)$ were recorded on the watch. The $10 \%$ of SIWPs that were missing on the watch were due to either the patient not pressing the watch or the patient pressing the button incorrectly when stopping due to ischemic pain.

Despite the $\sim 10 \%$ missing case rate in the recordings of the WPMs or SIWPs using the watch, because the PAD participants usually performed multiple walking bouts during their OWS, the PFWT could not be computed in only one patient (with no WPM). Comparing the times at which the WPMs and SIWPs were recorded on the watch and the time they were observed by the investigators, a mean difference of $4 \pm 12 \mathrm{~s}$ for the WPMs and $1 \pm 5 \mathrm{~s}$ for the SIWPs was found. This finding translated into nearly perfect correlation coefficients between 
the average PFWT computed from the watch and that computed from the observations $(\mathrm{r}=0.967, \mathrm{n}=19, \mathrm{P}<0.001)$ and between the average MWT computed from the watch and that computed from the observations $(\mathrm{r}=0.992, \mathrm{n}=21, \mathrm{P}<0.001)$.

Relationship with treadmill walking tests and questionnaires. Compared to the results of the Strandness treadmill test (Table 2), the PFWT ${ }^{[\mathrm{OWS}]}$ and the $\mathrm{MWT}^{[\mathrm{OWS}]}$ (Table 3 and Figure 2) were significantly higher $(\mathrm{P}<0.001)$. The differences were statistically significant compared to the $\mathrm{PFWT}^{[\mathrm{GARDNER}]}(\mathrm{P}=0.014)$ but not the $\mathrm{MWT}^{[\mathrm{GARDNER}]}(\mathrm{P}=0.297)$. The $\mathrm{PFWT}^{[\mathrm{OWS}]}$ and $\mathrm{MWT}^{[\mathrm{OWS}]}$ were significantly correlated with the Strandness PFWT $(\mathrm{r}=0.955, \mathrm{n}=17, \mathrm{P}<0.001)$ and MWT ( $r=0.821, \mathrm{n}=19, \mathrm{P}<0.001)$, respectively (Appendix \#1). Significant associations were also found between the $\mathrm{PFWT}^{[\mathrm{Gardner}]}$ and the $\mathrm{PFWT}^{[\mathrm{OWS}]}(\mathrm{r}=0.721, \mathrm{n}=18, \mathrm{P}=0.001)$ and between the $\mathrm{MWT}^{[\mathrm{Gardner}]}$ and the $\mathrm{MWT}^{[\mathrm{OWS}]}(\mathrm{r}=0.446, \mathrm{n}=21, \mathrm{P}=0.043)$, although the correlation coefficients were lower (Appendix \#1). The PFWT ${ }^{[\mathrm{OWS}]}$ and $\mathrm{MWT}^{[\mathrm{OWS}]}$ were also significantly correlated with the WIQ subscale and total scores and the physical functioning score computed from the SF-36 questionnaire (Appendix \#2). In addition, the physical component summary score was moderately correlated with the $\mathrm{PFWT}^{[\mathrm{OWS}]}(\mathrm{r}=0.467, \mathrm{n}=19$, $\mathrm{P}=0.044)$.

As shown in Appendices \#1 and \#2, one patient had a higher walking capacity than the remaining PAD participants. Removing this patient from the correlation analyses only had an effect on the correlations with the MWT ${ }^{[\text {Gardner] }}$, the $\mathrm{WIQ}^{[\text {Speed] }}$ subscale scores and the SF36 scores (the correlations were no longer significant).

\section{7-day free-living measurement}

Acceptability and compliance with the use of the wearable monitors. Four and two participants reported experiencing discomfort from the watch (wrist skin irritation) and belt of the wGT3X+, respectively. Another patient reported that the wearable monitors were 
perceived as psychologically disturbing (feeling that he/she was being continuously monitored). Three participants reported that they did not systematically press the event marker button of the watch to indicate WPMs. One patient only wore the wGT3x+ for 2 days and the watch for 4 days of the 7 days and was excluded from subsequent analyses. Thus, among the expected total of 161 days of recording (7 days $\times 23$ participants), 150 (93\%) days were considered valid for the watch and $148(92 \%)$ days were considered valid for the wGT3X+; of these days, 146 days were valid for both monitors. All remaining participants included in the analysis $(\mathrm{n}=22)$ had at least five valid days of data from the two wearable monitors, including at least one weekend day, and 73\% (16/22) of the participants had seven valid days of data from both wearable monitors.

Lower limb pain manifestations and associated functional limitations during daily life walking.

Among the 146 valid days available for analysis, 107 days were associated with one or more event markers, leading to a total of 670 events among the 22 participants. Among the 670 event markers recorded, 55 (8\%) events were labeled "inconsistent". These "inconsistent" events occurred either at random during a resting period far from the stopping point of a walking bout $(n=51)$ or, more rarely, at the beginning of a walk $(n=4)$.

After removing duplicate pushes related to pain or stop events, the final number of WPMs and SIWPs collected among the 22 participants was 368, and high heterogeneity was observed among the PAD participants (range: 0-49/week, see Figure 3). No WPMs or SIWPs were reported in one patient, and only four of the 22 participants experienced WPMs or SIWPs on a daily basis. As shown in Table 4, overall, the PAD participants experienced a low number of WPM or SIWP events during the seven days of the free-living recording. The PAD participants experienced many more WPMs than SIWPs, and only $16 \%$ of the WPMs led to SIWPs. Considering only the number of valid days at the group level, on average, the 
PAD participants experienced a WPM only twice per day and an SIWP only once a day (Table 4). As shown in Table 4, this group of PAD participants spent 64\% [52-72] of their daily time engaging in sedentary behavior and $8 \%$ [5-15] of their time engaging in moderate to vigorous physical activity. Furthermore, the number of bouts $\geq 10$ minutes with a string of consecutive counts values $\geq 760$ counts/minute was very low, i.e., 2 [1-8]. Using the notebook, the context in which each event related to WPMs or SIWPs occurred could be identified for 288 of 368 events (78\%) because some participants sometimes did not complete the diary properly. Most (72\%) WPM or SIWP events occurred while the participants were outside their home.

When calculating the 7-day FLM maximal PFWT from the WPMs and the maximal MWT from the SIWPs, we found that PFWT ${ }^{[\mathrm{FLM}]}$ and $\mathrm{MWT}^{[\mathrm{FLM}]}$ were on average 6 times higher than $\mathrm{PFWT}^{[\mathrm{OWS}]}(\mathrm{P}<0.001)$ and 3 times higher than $\mathrm{MWT}^{[\mathrm{OWS}]}(\mathrm{P}=0.009)$. In addition, the $\mathrm{PFWT}^{[\mathrm{FLM}]}$ was on average 12 times longer than the PFWT ${ }^{[\text {Strandness] }}$ (Figure 2 and Table 4). The related intensity of the walking bouts as assessed by the VM mean counts was also significantly lower during the FLM than that during the OWS ( $45 \pm 15$ vs. $66 \pm 21$ counts/s, $\mathrm{P}<0.001$ ). Moderate but significant inverse correlations (Appendix \#3) were found between the mean WPM/day and the MWT ${ }^{[\mathrm{GARDNER}]}(\mathrm{r}=-0.472, \mathrm{n}=22, \mathrm{P}=0.027)$, the $\mathrm{PFWT}^{[\text {Strandness }]}$ $(\mathrm{r}=-0.543, \mathrm{n}=19, \mathrm{P}=0.016)$, the MWT ${ }^{[\text {Strandness }]}(\mathrm{r}=-0.527, \mathrm{n}=20, \mathrm{P}=0.017)$, the $\mathrm{PFWT}^{[\mathrm{OWS}]}(\mathrm{r}=-$ $0.473, \mathrm{n}=19, \mathrm{P}=0.041)$, and the $\mathrm{WIQ}^{[\text {Distance] }}$ subscale score $(\mathrm{r}=-0.438, \mathrm{n}=22, \mathrm{P}=0.041)$. However, these correlations should be interpreted with caution considering the low number of WPMs/day experienced by the PAD participants. The very low number of SIWPs experienced by the participants precluded any robust correlation analysis. 


\section{DISCUSSION}

To the best of our knowledge, this study is the first to propose an event-based analysis method using a combination of wearable monitors to identify and quantify lower limb WPMs and associated functional limitations (SIWPs) in PAD participants while walking under freeliving conditions.

The main results can be summarized as follows: (i) the use of the watch to report WPMs or SIWPs during a 45 to 65 -minute OWS showed a high feasibility with only a $10 \%$ missing data rate; (ii) both the PFWT and the MWT computed from the identification of WPMs and SIWPs using the watch during the OWS were significantly correlated with the PFWT ${ }^{[\text {Strandness }]}$ and $\mathrm{MWT}^{[\text {Strandness }]}$; and (iii) although severely limited with regard to walking on a treadmill and during the OWSs, this cohort of PAD participants who engaged in very few continuous bouts of moderate-to-vigorous physical activity experienced a very low number of events related to WPMs or SIWPs during their daily life.

The walking capacity under daily life conditions in PAD participants remains largely understudied and, thus, poorly understood. In the present study, we focused on two different types of measurements that can provide complementary information to clinicians assessing the pain experienced by people with PAD under daily walking conditions.

\section{PFWT and MWT during an outdoor walking session}

The design of the OWS used in the present study was similar to that used in other studies assessing the outdoor walking capacity ${ }^{6,7,15,16}$ and is very similar to the design of walking sessions performed during home-based walking programs ${ }^{31}$. To the best of our knowledge, this study is the first to assess PFWT during outdoor walking in PAD participants. Using a GPS receiver, previous studies have reported a significant correlation between the MWT (or distance) during an outdoor walk and a treadmill-walking test in PAD participants ${ }^{6,7}$. Our 
results and the method proposed here extend the possibilities offered by wearable monitors in assessing outdoor walking capacity in PAD participants ${ }^{11}$.

First, by assessing the "natural" PFWT in addition to the MWT during outdoor walking, clinicians can fully assess the pain experienced by PAD participants for determining the baseline walking capacity and exploring the therapeutic effects of interventions. As previously reported regarding the maximal walking distance/time $e^{7,15,16}$, we found that the PFWT during outdoor walking was significantly higher (by a factor of 3 ) than the PFWT measured during the Strandness treadmill test. This substantial variation supports the fact that participants rightly consider treadmill assessments a poor reflection of their walking disability 9 .

Second, the identification of SIWPs could improve the clinical interpretation of unsupervised outdoor walking capacity assessments. Indeed, by discriminating walking bouts that induce SIWPs during an OWS from those that do not, the risk of excluding participants who reported not stopping only because of leg-related symptoms could be considerably lowered ${ }^{7,16}$

From a clinical utility perspective, the use of physical activity monitors has the potential of improving the clinical management of people with chronic conditions ${ }^{32}$. In the present case, and although future studies should be performed, an outdoor assessment of PFWT (and MWT) using physical activity monitors could contribute to the better clinical management of PAD people for both diagnostic and therapeutic purposes as follows:

- By complementing (not replacing) standardized treadmill evaluations to finely assess the walking capacity of people with PAD under a context of "natural" walking and identify potential changes following interventions.

- By providing a way to objectively assess the walking capacity of PAD people when treadmill assessments are not available. 
- By implementing efficient home-based walking programs with continuous monitoring of walking capacity outcomes using wearable monitors during outdoor walking sessions.

\section{Daily life WPM in PAD}

While a prescribed OWS is a means of assessing the "natural" walking capacity under semicontrolled walking conditions, the aim of the 7-day FLM period was to explore the pain experienced by PAD participants in the daily life context.

Methodological considerations. To develop our event-based analysis method to analyze the FLM period, one of the main methodological challenges for identifying WPMs and SIWPs from wearable monitor data was to discriminate walking bouts from non-walking bouts. Methodologies have been proposed in previous studies to discriminate walking from other behaviors, but these methodologies implement discrimination thresholds at the minute level ${ }^{33}$. In our study, because walking pain could occur at random in PAD participants, it was crucial for our application to use a higher time resolution than that proposed in these studies. The use of a combination of two wearable monitors to label each event marker raised the issue of between-monitor synchronization. The preliminary tests performed prior to our study showed that the maximal time difference between the wGT3X+ monitor and the watch at the end of the 7-day period was $15 \mathrm{~s}$ (data not shown). Such a time difference was consistent with our labeling methodology and the use of a minimum duration of $15 \mathrm{~s}$ to define walking and non-walking bouts.

Clinical interpretation. We found that on average, few events related to WPMs or SIWPs occurred on a daily basis in the PAD participants. Initially, this result could appear quite surprising considering both the treadmill and OWS results in our population and the high 
level of disability reported by PAD participants in their daily life due to walking pain as demonstrated by the poor WIQ scores.

Our population of PAD participants engaged in very few bouts of continuous moderate-tovigorous physical activity (see Table 4). Thus, although no causal inference can be drawn from our data, it could be suggested that such a pattern of daily physical activity prevents the occurrence of lower limb pain during walking in PAD participants. It is acknowledged that walking-induced pain is among the most frequent barriers to engaging in walking reported by PAD participants ${ }^{18}$. Thus, clinically, the low level of WPMs or SIWPs reported here during the FLM periods basically show that the participants may be avoiding activity rather than that the participants were not functionally limited. Furthermore, although unlikely at the sample level and over the entire duration of the recording, it cannot be completely ruled out that weather conditions could have influenced the pattern of (outdoor) physical activity during the FLM period and, in turn, the occurrence of WPM.

Independently or in conjunction with the pattern of daily physical activity, we found that the intensity of free-living walking as measured by the mean VM was lower than the walking intensity during the OWS. This finding could explain why the participants did not report a large number of WPM events.

Taken together, our results support the well-known discrepancy between the leg-related symptoms reported (or not) by symptomatic (or asymptomatic) PAD participants and the actual impairment of their leg function when objectively assessed ${ }^{34}$. A conjunction of factors likely explains the pattern of symptom occurrence during daily life in PAD participants. Clinicians should consider that a joint and objective assessment of physical activity and sedentary behaviors is important to fully assess the pain experienced by PAD participants under free-living conditions. 
From a clinical utility perspective, qualitative studies have documented that living with PAD is associated with pain in daily life, which impairs and limits mobility ${ }^{35}$. Consequently, physical and social functions are negatively affected, leading to a decreased quality of life ${ }^{35}$. However, other than the knowledge provided by qualitative studies, no direct information is available regarding the pattern of walking pain in daily life, i.e., on a day-to-day basis. When and how often does ischemic pain occurs? Which type, duration and intensity of daily life activities are mainly "at risk" of causing ischemic pain? Does pain occurrence lead people with PAD to stop walking in all cases? Our opinion is that such information could help patients to better manage their "pain experience" and engage in daily walking. The method proposed here opens interesting opportunities to address these issues.

\section{Study limitations}

Some limitations of the present study should be acknowledged. First, although the missing data rate in the reporting of the WPMs or SIWPs during the OWS was low and the monitorwearing compliance during the 7-days of FLM was high, some participants highlighted that the watch should be more ergonomic to facilitate pressing the event marker button. This feedback may explain the high number of multiple presses performed by the participants to ensure the recording of the events. In the present study, we used a watch as an event recorder because the recording of event markers over a long period of time was feasible ( $>7$ days at a 5-s recording rate). To the best of our knowledge, no other technical solution was commercially available when we designed the present study.

Second, we identified that the events related to WPMs or SIWPs during the seven days of FLM mainly occurred during continuous outside activities. Although this result seems to be consistent with the fact that a prolonged bout of walking could more likely occur when walking outside, it should be interpreted with caution because some timestamp distortions 
could appear when the daily activities were recalled at bedtime and recorded by the participants in their diary. Adding a GPS receiver could have helped in performing this discrimination and could have increased the accuracy of our analysis of the FLM results. The use of a GPS receiver could add relevant information related to WPMs or SIWPs, such as the location (environmental context) and the indoor/outdoor context, the speed of the outdoor walk and the distance walked when it occurs. In the present study, the choice was made to not add a GPS receiver during the seven days of FLM to limit the burden on the participants due to wearing multiple physical activity monitors. Furthermore, the DG100 GPS receiver is not suitable for prolonged measurements, and the combination of the watch and a GPS receiver (instead of the accelerometer) could have precluded the analysis of any WPMs or SIWPs occurring indoors.

Third, it is unknown whether, at the patient level, the experience of recording pain affects the experience of pain. This issue deserves further study. Finally, the VM count threshold used was derived from a previous study in which the authors applied this threshold to discriminate sedentary behaviors from physical activity ${ }^{28}$. To the best of our knowledge, no study has proposed a specific count threshold to discriminate walking bouts from non-walking bouts at the second level. Although the counts threshold used in the present study appeared relevant when implementing our algorithm, future studies should refine this method.

\section{Future directions}

A first and important technical step could be to design a more suitable and ergonomic watch to facilitate the task for the participants when reporting events related to a WPM or SIWP.

New technical solutions are currently under development (SHERPAM project: https://sherpam.cominlabs.ubretagneloire.fr/fr/aomi;jsessionid=FFDDF78A949065BC26AADFA3CC17C2AB). Future studies should also determine the interest in using or adding other types of wearable 
monitors, such as a GPS receiver as explained above. Furthermore, the ActivPAL ${ }^{\mathrm{TM}}$ monitor could potentially facilitate the discrimination of marker events (WPMs and SIWPs), and the methodology proposed by Clarke et al. ${ }^{17}$ should be considered in light of that proposed here. We acknowledge that our methodology should be applied and tested in another population of PAD participants. We are confident about the external validity of this methodology because it did not appear to be patient-specific. The next step is to design cross-sectional studies involving a large sample of PAD participants to investigate the complex interactions among lower limb symptom occurrence during daily life, walking capacity, physical activity patterns and avoidance, and quality of life.

\section{PERSPECTIVE}

The present study proposes a new method using wearable monitors to objectively identify and quantify lower limb WPMs and SIWPs during OWSs and daily life in a sample of PAD participants. The PFWT and the MWT derived from this method were significantly correlated with treadmill measures of walking capacity. Assessing events related to WPMs or SIWPs in daily life can provide researchers and clinicians a more realistic and holistic view of the experience of living with PAD (burden of the disease) and the associated functional limitations and, consequently, could be a promising clinical and research tool for PAD management. 


\section{CONFLICTS OF INTEREST}

None

\section{ACKNOWLEDGMENTS}

The authors are indebted to Miss Hala Abdul Rahman for her technical help in implementing the processing methodology.

\section{SOURCES OF FUNDING}

This work was partially supported by the University Hospital of Rennes (CORECT 2013). S. Chaudru received grants from the Bretagne region (Bourse ARED) and the SFETD/APICIL foundation. P.-Y. de Müllenheim received a PhD scholarship from the École normale supérieure de Cachan-Antenne de Bretagne (CDSN).

\section{NOTE}

Pierre-Yves de Müllenheim is currently working at the Institute of Physical Education and Sport Sciences (IFEPSA) (West Catholic University, UCO, Les Ponts-de-Cé, France). 


\section{REFERENCES}

1. McDermott MM. Lower extremity manifestations of peripheral artery disease: the pathophysiologic and functional implications of leg ischemia. Circ Res.

2015;116(9):1540-1550.

2. Hirsch AT, Haskal ZJ, Hertzer NR, et al. ACC/AHA 2005 Practice Guidelines for the management of patients with peripheral arterial disease (lower extremity, renal, mesenteric, and abdominal aortic): a collaborative report from the American Association for Vascular Surgery/Society for Vascular Surgery, Society for Cardiovascular Angiography and Interventions, Society for Vascular Medicine and Biology, Society of Interventional Radiology, and the ACC/AHA Task Force on Practice Guidelines (Writing Committee to Develop Guidelines for the Management of Patients With Peripheral Arterial Disease): endorsed by the American Association of Cardiovascular and Pulmonary Rehabilitation; National Heart, Lung, and Blood Institute; Society for Vascular Nursing; TransAtlantic Inter-Society Consensus; and Vascular Disease Foundation. Circulation. 2006;113(11):e463-654.

3. Nicolai SP, Viechtbauer W, Kruidenier LM, Candel MJ, Prins MH, Teijink JA. Reliability of treadmill testing in peripheral arterial disease: a meta-regression analysis. J Vasc Surg. 2009;50(2):322-329.

4. Treat-Jacobson D, Henly SJ, Bronas UG, Leon AS, Henly GA. The pain trajectory during treadmill testing in peripheral artery disease. Nurs Res. 2011;60(3 Suppl):S3849.

5. Seenan C, McSwiggan S, Roche PA, Tan CW, Mercer T, Belch JJ. Transcutaneous Electrical Nerve Stimulation Improves Walking Performance in Patients With Intermittent Claudication. J Cardiovasc Nurs. 2016;31(4):323-330.

6. Fokkenrood HJ, van den Houten MM, Houterman S, Breek JC, Scheltinga MR, Teijink JA. Agreements and discrepancies between the estimated walking distance, nongraded and graded treadmill testing, and outside walking in patients with intermittent claudication. Ann Vasc Surg. 2015;29(6):1218-1224.

7. Le Faucheur A, Abraham P, Jaquinandi V, Bouye P, Saumet JL, Noury-Desvaux B. Measurement of walking distance and speed in patients with peripheral arterial disease: a novel method using a global positioning system. Circulation. 2008;117(7):897-904.

8. Watson CJ, Collin J. Estimates of distance by claudicants and vascular surgeons are inherently unreliable. Eur J Vasc Endovasc Surg. 1998;16(5):429-430.

9. Barletta G, Perna S, Sabba C, Catalano A, O'Boyle C, Brevetti G. Quality of life in patients with intermittent claudication: relationship with laboratory exercise performance. Vasc Med. 1996;1(1):3-7.

10. Frans FA, Zagers MB, Jens S, Bipat S, Reekers JA, Koelemay MJ. The relationship of walking distances estimated by the patient, on the corridor and on a treadmill, and the Walking Impairment Questionnaire in intermittent claudication. J Vasc Surg. 2013;57(3):720-727 e721.

11. de Mullenheim PY, Chaudru S, Mahe G, Prioux J, Le Faucheur A. Clinical Interest of Ambulatory Assessment of Physical Activity and Walking Capacity in Peripheral Artery Disease. Scand J Med Sci Sports. 2016;26(7):716-730.

12. Hiatt WR, Rogers RK, Brass EP. The Treadmill Is a Better Functional Test Than the 6-Minute Walk Test in Therapeutic Trials of Patients With Peripheral Artery Disease. Circulation. 2014;130(1):69-78.

13. Le Faucheur A, de Mullenheim PY, Mahe G. Letter by Le Faucheur et al regarding articles, "Six-minute walk is a better outcome measure than treadmill walking tests in 
therapeutic trials of patients with peripheral artery disease" and "The treadmill is a better functional test than the 6-minute walk test in therapeutic trials of patients with peripheral artery disease". Circulation. 2015;131(15):e406.

14. McDermott MM, Guralnik JM, Criqui MH, Liu K, Kibbe MR, Ferrucci L. Six-Minute Walk Is a Better Outcome Measure Than Treadmill Walking Tests in Therapeutic Trials of Patients With Peripheral Artery Disease. Circulation. 2014;130(1):61-68.

15. Gernigon M, Le Faucheur A, Noury-Desvaux Bnd, Mahe G, Abraham P, Post GPSSCG. Applicability of global positioning system for the assessment of walking ability in patients with arterial claudication. Journal of Vascular Surgery. 2014;60(4):973-981.e971.

16. Le Faucheur A, Noury-Desvaux B, Mahe G, et al. Variability and short-term determinants of walking capacity in patients with intermittent claudication. $J$ Vasc Surg. 2010;51(4):886-892.

17. Clarke CL, Holdsworth RJ, Ryan CG, Granat MH. Free-living physical activity as a novel outcome measure in patients with intermittent claudication. Eur J Vasc Endovasc Surg. 2013;45(2):162-167.

18. Abaraogu U, Ezenwankwo E, Dall P, et al. Barriers and enablers to walking in individuals with intermittent claudication: A systematic review to conceptualize a relevant and patient-centered program. PLoS One. 2018;13(7):e0201095.

19. Criqui MH, Denenberg JO, Bird CE, Fronek A, Klauber MR, Langer RD. The correlation between symptoms and non-invasive test results in patients referred for peripheral arterial disease testing. Vasc Med. 1996;1(1):65-71.

20. McDermott MM, Greenland P, Liu K, et al. Leg symptoms in peripheral arterial disease: associated clinical characteristics and functional impairment. Jama. 2001;286(13):1599-1606.

21. Aboyans V, Criqui MH, Abraham P, et al. Measurement and interpretation of the ankle-brachial index: a scientific statement from the American Heart Association. Circulation. 2012;126(24):2890-2909.

22. Hiatt WR, Hirsch AT, Regensteiner JG, Brass EP. Clinical trials for claudication. Assessment of exercise performance, functional status, and clinical end points. Vascular Clinical Trialists. Circulation. 1995;92(3):614-621.

23. Leplege A, Ecosse E, Verdier A, Perneger TV. The French SF-36 Health Survey: translation, cultural adaptation and preliminary psychometric evaluation. J Clin Epidemiol. 1998;51(11):1013-1023.

24. Mahe G, Ouedraogo N, Vasseur M, et al. Limitations of self-reported estimates of functional capacity using the Walking Impairment Questionnaire. Eur J Vasc Endovasc Surg. 2011;41(1):104-109.

25. McDermott MM, Liu K, Guralnik JM, Martin GJ, Criqui MH, Greenland P. Measurement of walking endurance and walking velocity with questionnaire: validation of the walking impairment questionnaire in men and women with peripheral arterial disease. J Vasc Surg. 1998;28(6):1072-1081.

26. Le Faucheur A, Abraham P, Jaquinandi V, Bouy ̃̃@ P, Saumet JL, Noury-Desvaux BND. Study of Human Outdoor Walking with a Low-Cost GPS and Simple Spreadsheet Analysis. Medicine \& Science in Sports \& Exercise. 2007;39(9):15701578.

27. Choi L, Liu Z, Matthews CE, Buchowski MS. Validation of accelerometer wear and nonwear time classification algorithm. Med Sci Sports Exerc. 2011;43(2):357-364.

28. Aguilar-Farías N, Brown WJ, Peeters GMEE. ActiGraph GT3X+ cut-points for identifying sedentary behaviour in older adults in free-living environments. Journal of Science and Medicine in Sport. 2014;17(3):293-299. 
29. Ainsworth BE, Haskell WL, Herrmann SD, et al. 2011 Compendium of Physical Activities: A Second Update of Codes and MET Values. Medicine \& Science in Sports \& Exercise. 2011;43(8):1575-1581.

30. Matthew CE. Calibration of accelerometer output for adults. Med Sci Sports Exerc. 2005;37(11 Suppl):S512-522.

31. Gardner AW, Parker DE, Montgomery PS, Scott KJ, Blevins SM. Efficacy of quantified home-based exercise and supervised exercise in patients with intermittent claudication: a randomized controlled trial. Circulation. 2011;123(5):491-498.

32. Chow CK, Ariyarathna N, Islam SM, Thiagalingam A, Redfern J. mHealth in Cardiovascular Health Care. Heart Lung Circ. 2016;25(8):802-807.

33. Migueles JH, Cadenas-Sanchez C, Ekelund U, et al. Accelerometer Data Collection and Processing Criteria to Assess Physical Activity and Other Outcomes: A Systematic Review and Practical Considerations. Sports Med. 2017;47(9):1821-1845.

34. McDermott MM. Functional impairment in peripheral artery disease and how to improve it in 2013. Curr Cardiol Rep. 2013;15(4):347.

35. Egberg L, Andreassen S, Mattiasson A-C. Experiences of living with intermittent claudication. Journal of Vascular Nursing: Official Publication of the Society for Peripheral Vascular Nursing. 2012;30(1):5-10. 


\section{FIGURES LEGENDS}

Figure 1. Left panel: Schematic representation of the wearable monitor-based methodology developed to label each event marker recorded by the watch during the 7-day free-living measurement. Right panel: A typical example of the event identification performed in one patient.

\section{Legend}

VM: Vector magnitude. WPM: Walking pain manifestation. SIWP: Stop induced by walking pain.

Left panel. The first step was to discriminate walking bouts from non-walking bouts using the VM count data recorded by the wGT3X+. This approach consisted of using a counts/s threshold as previously proposed to identify sedentary behavior in adults in free-living environments ${ }^{28}$. Our rationale was as follows: a string of consecutive count data with a VM $\geq 1$ count/s for $\geq 15 \mathrm{~s}$ was defined as a walking bout and that with a VM=0 count/s for $\geq 15 \mathrm{~s}$ was defined as a non-walking bout. A string of consecutive count data with a VM $\geq 1$ counts/s for $<15 \mathrm{~s}$ was converted to a non-walking bout. Conversely, a string of consecutive counts data with a $\mathrm{VM}=0$ count/s for $<15 \mathrm{~s}$ was converted to a walking bout. The rationale of using a minimum duration of $15 \mathrm{~s}$ to define the walking and non-walking bouts was that walkinginduced ischemia was unlikely to appear (during walking) or disappear (during stopping) within such a short period ${ }^{15}$. An event marker was labeled a WPM when it occurred first during a walking bout and at least $\geq 15 \mathrm{~s}$ after the beginning of the walking bout. A single or a double event marker was labeled as an SIWP when the marker occurred during the first 15 seconds of a stopping bout that followed a walking bout or during the last 15 seconds of a walking bout immediately before a stopping bout. 
Right panel. The upper right panel (A) shows the VM counts over time measured throughout an entire day (24 hours) in one patient. The events related to walking pain manifestation (WPM) and stop induced by walking pain (SIWP) recorded and processed using our methodology are also displayed on the graph. The color bars indicate when the patient was at home or outside on the basis of the information reported in the notebook. In the lower right panel (B), the time period during which a WPM or SIWP appeared (during this day in this patient) is presented.

Figure 2. Pain free-walking times and maximal walking times in each context of measurement in PAD participants.

\section{Legend}

PFWT: Pain-free walking time. MWT: Maximal walking time. OWS: Outdoor walking session. FLM: 7-day free-living measurement.

a: $\mathrm{P}<0.05$ vs. Strandness test; $\mathrm{b}: \mathrm{P}<0.05$ vs. Gardner test; c: $\mathrm{P}<0.05$ vs. outdoor walking session; $\mathrm{d}$ : $\mathrm{P}<0.05$ vs. the 7-day free-living measurement period.

Figure 3. Overview of the number of events related to lower limb pain manifestation and stop induced by walking pain per PAD patient.

\section{Legend}

WPM: Walking pain manifestation. SIWP: Stop induced by walking pain. 


\section{APPENDICES LEGENDS}

Appendix \#1. Relationship between the walking capacity measured during the outdoor walking session and that measured during the treadmill tests.

\section{Legend}

PFWT: Pain-free walking time. MWT: Maximal walking time. OWS: Outdoor walking session.

Appendix \#2. Relationship between the walking capacity measured during the outdoor walking session and the scores obtained from the walking impairment questionnaire and the SF-36 questionnaire.

\section{Legend}

PFWT: Pain-free walking time. MWT: Maximal walking time. OWS: Outdoor walking session. WIQ: Walking impairment questionnaire. PFs: Physical functioning score.

Appendix \#3. Relationship between the average number of daily walking pain manifestationrelated events as assessed during the 7-day free-living measurement and some walking capacity measures.

\section{Legend}

PFWT: Pain-free walking time. MWT: Maximal walking time. WPM: Walking pain manifestation. OWS: Outdoor walking session. WIQ: Walking impairment questionnaire. 
Table 1. Inclusion and non-inclusion criteria of the CLASH study.

\section{Inclusion criteria}

- $\quad$ Age $\geq 18$ years old

- Insured under the French social security system (according to French law)

- Presence of lower-extremity peripheral artery disease, defined by:

- A resting ankle-brachial index $(\mathrm{ABI}) \leq 0.90$

$\circ \mathbf{O R}$ if resting $\mathrm{ABI}>0.90$ and $<1.00$, a decrease in recovery ankle systolic pressure or in recovery ABI from treadmill exercise higher than $30 \%$ or $20 \%$, respectively (AHA recommendations).

$\circ \quad$ OR if resting $\mathrm{ABI}>1.40$, a toe pressure index $\leq 0.70$

- Maximal walking distance on treadmill $(3.2 \mathrm{~km} / \mathrm{h}, 10 \%$ grade $)<500 \mathrm{~m}^{\mathrm{a}}$

- Complain of exertional lower limbs pain that sometimes can begin at rest, causes the participant to stop walking and relieves or lessens within 10 minutes of rest (assessed using the San Diego questionnaire AND confirmed during treadmill testing) ${ }^{\text {b }}$

\section{Non-inclusion criteria}

- Exercise limitation due to symptoms not related to an arterial insufficiency in the lower limbs (e.g., dyspnea, angina pectoris)

- Contraindication for walking (Abdominal aortic aneurysm $>4 \mathrm{~cm}$ )

- Myocardial infarction and no stroke in the last 3 months

- Critical limb ischemia, amputation.

- Pregnant women

- Adult subject to legal protection (guardianship or tutelage measure) and persons deprived from their liberty (according to French law).

- Patient living more than $50 \mathrm{~km}$ from the university hospital

- Patient unable to understand the instructions of the study 
${ }^{a}$ As assessed during the medical appointment.

${ }^{\mathrm{b}}$ According to our inclusion criteria, PAD participants' leg symptoms that fell within the following leg symptom categories ${ }^{13}$ could be included in the study:

i) Intermittent claudication. Patients that experience exertional calf pain that does not begin at rest and that forces them to stop walking and that relieves or lessens within 10 minutes of rest;

ii) Atypical exertional leg pain/stop. This category can encompass diverse situations of exertional leg symptoms. In the present study, PAD participants in this category were included if they experience exertional pain that does not begin at rest and that forces them to stop walking, but that do not involve only the calf(s) but also thigh(s) and/or buttock(s). Further, the exertional leg pain relieves or lessens within 10 minutes of rest;

iii) Leg pain on exertion and rest. In this category, people with PAD experience exertional lower limbs pain during walking but sometimes this pain can begin at rest when they are standing still or sitting. As reminded by Criqui et al. (see reference \#19), this category of people with PAD with "pain at rest" should not be confused with those that experience "rest pain", which usually refers to people with such severe advanced PAD that ischemic pain is present even at rest. Subjects with ischemic rest pain were not included in the study. 
Table 2. Characteristics of included peripheral arterial disease participants

General

Age, y

$60 \pm 10$

Male sex, n (\%)

$20(87)$

Stature, $\mathrm{cm}$

$169 \pm 8$

Body mass, $\mathrm{kg}$

$78.9 \pm 16.3$

Body mass index, $\mathrm{kg} / \mathrm{m}^{2}$

$27.4 \pm 4.7$

Ankle-brachial index

$0.61[0.53 ; 0.75]$

Leg symptoms reported / assessed on treadmill', $n(\%)$

Intermittent claudication Rose

$17(74) / 18(78)$

Leg pain on exertion and rest

$5(22) / 5(22)$

Atypical claudication-carry on

$1(4) / 0(0)$

Comorbid conditions, $n(\%)$

Current smoker

Former smoker

$23(100)$

Previous lower-extremity revascularization

History of myocardial infarction

Chronic bronchitis

$6(26)$

Diabetes mellitus

$6(26)$

Medications used, $n(\%)$

Antiplatelet agents

$18(78)$

Statins

Anti-diabetics

Vasodilators

$\beta$-blockers 
Antihypertensive drugs

$14(61)$

Treadmill walking capacity assessment

Gardner-Skinner treadmill test

Pain-free walking time, $\mathrm{s}$

$131[89 ; 224]$

Maximal walking time, $\mathrm{s}$

$289[203 ; 420]$

Strandness treadmill test

Pain-free walking time, $\mathrm{s}$

$79[56 ; 105]$

Maximal walking time, $\mathrm{s}$

178 [116;213]

\section{Questionnaires}

Short-form 36

Physical functioning score (PFs)

$50[35 ; 70]$

Role-physical score (RPs)

$25[0 ; 100]$

Bodily pain score (BPs)

$41[31 ; 42]$

General health score (GHs)

$52[30 ; 62]$

Vitality score (VTs)

$40[25 ; 60]$

Social functioning score (SFs)

$63[38 ; 88]$

Role-emotional score (REs)

$33[0 ; 100]$

Mental health (MHs)

$68[52 ; 84]$

Physical component summary score (PCS)

$34[29 ; 43]$

Mental component summary score (MCS)

$42[37 ; 55]$

Walking impairment questionnaire

Distance subscale score

$20[11 ; 40]$

Speed subscale score

$25[17 ; 33]$

Stairs climbing subscale score

$42[21 ; 75]$

Total score

$34[17 ; 49]$ 
Continuous variables are expressed as medians with interquartile range [IQR $=25^{\text {th }} ; 75$ th percentiles] or as mean \pm SD. Data for categorical variables are expressed as a number (percentage).

$\dagger$ Leg symptoms reported using the San Diego claudication questionnaire and then assessed during the treadmill tests; See Table 1 for definition. Atypical claudication-carry on category refers to PAD participants that experience exertional leg symptoms that do not begin at rest and that do not stop them from walking. 
Table 3. Results from the outdoor walking session.

\section{Atmospheric conditions (mean \pm SD)}

Temperature, ${ }^{\circ} \mathrm{C}$

$15 \pm 5$

Relative humidity, \%

$68 \pm 15$

Atmospheric pressure, $\mathrm{hPa}$

$1020 \pm 10$

Wind speed, $\mathrm{km} / \mathrm{h}$

$15 \pm 8$

\section{GPS derived-parameters}

Median [IQR] duration of the session, min

Mean \pm SD total distance, $\mathrm{m}$

Mean \pm SD walking speed, $\mathrm{km} / \mathrm{h}$

$4.2 \pm 0.4$

Median [IQR] number of stops

$6[4 ; 10]$

Median [IQR] MWT, s

- All walking bouts

$290[164 ; 358]$

- Longer walking bout

$329[215 ; 481]$

Median [IQR] duration of stops, min

$1.72[1.38 ; 3.00]$

\section{Accelerometer counts}

Mean \pm SD VM, counts/s

$64.9 \pm 20.4$

\section{Observation-derived parameters}

WPM events, $\mathrm{n}$

SIWP events, $\mathrm{n}$

170

Median [IQR] PFWT, s

- All walking bouts

$141[62 ; 217]$

- Longer walking bout

$187[89 ; 264]$

Median [IQR] MWT, s 
- All walking bouts

- Longer walking bout

\section{MML derived-parameters}

WPM events, $\mathrm{n}$

SIWP events, $\mathrm{n}$

Median [IQR] PFWT, s

- All walking bouts

$163[66 ; 220]$

- Longer walking bout

Median [IQR] MWT, s

- All walking bouts

- Longer walking bout

SD: Standard deviation. IQR: Interquartile range. GPS: Global positioning system. MML: Micro Motionlogger ${ }^{\circledR}$ Watch. VM: Vector magnitude. WPM: Walking pain manifestation. SIWP: Stop induced by walking pain. PFWT: Pain free walking time. MWT: Maximal walking time. 
Table 4. Results from the seven days free-living measurement.

$\begin{array}{lc}\text { WPM } & 282 \\ \text { Total number } & 13 \pm 10(0-30) \\ \text { Mean } \pm \text { SD event/week (range) } & 2 \pm 1(0-5) \\ \text { Mean } \pm \text { SD event/day (range) } & \\ \text { Median [IQR] PFWT, sec } & 342[272 ; 434] \\ \text { All walking bouts } & 778[526 ; 1163] \\ \text { Longer walking bout } & 46 \pm 15\end{array}$

\section{SIWP}

Total number

Mean \pm SD event/week (range)

Mean \pm SD event/day (range)

Median [IQR] PFWT, sec

All walking bouts

Longer walking bout

$888[505 ; 1333]$

Mean \pm SD VM

$43 \pm 19$

\section{Physical activity level}

Median [IQR] Time spent, min/d

Sedentary behaviors (SB)

$535[442-608]$

Light physical activity (LPA)

$234[172-282]$

Moderate physical activity (MPA)

$62[47-121]$

Vigorous physical activity (VPA)

0 [0-0] 
Median [IQR] Percent of time, \%/d

Sedentary behaviors (SB)

$64[52-72]$

Light physical activity (LPA)

$27[22-33]$

Moderate physical activity (MPA)

$8[5-15]$

Vigorous physical activity (VPA)

$0[0-0]$

Median $[\mathrm{IQR}]$ number of bouts $\geq 10 \mathrm{~min}$ and $\geq 760$ counts/min, $\mathrm{n}$

$2[1-8]$

WPM: Walking pain manifestation; SIWP: Stop induced by walking pain. VM: Vector magnitude.

SD: Standard deviation; IQR: Interquartile range

SB level: counts/min< 100; LPA level: counts/min between 100 and 759; MPA level: counts/min between 760 and 5724; VPA level: counts/min>5724 


\section{Figure \#1}

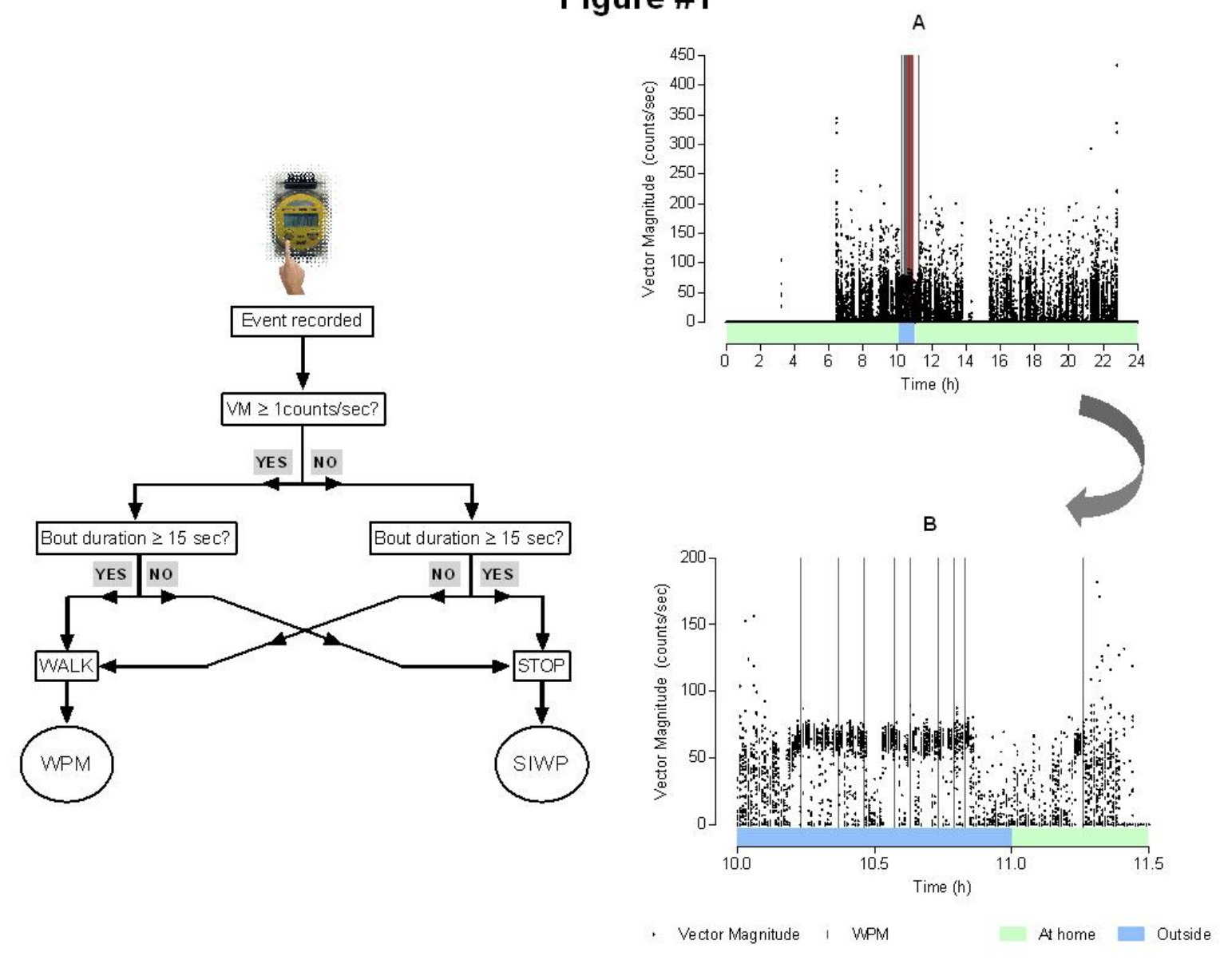


Figure \#2

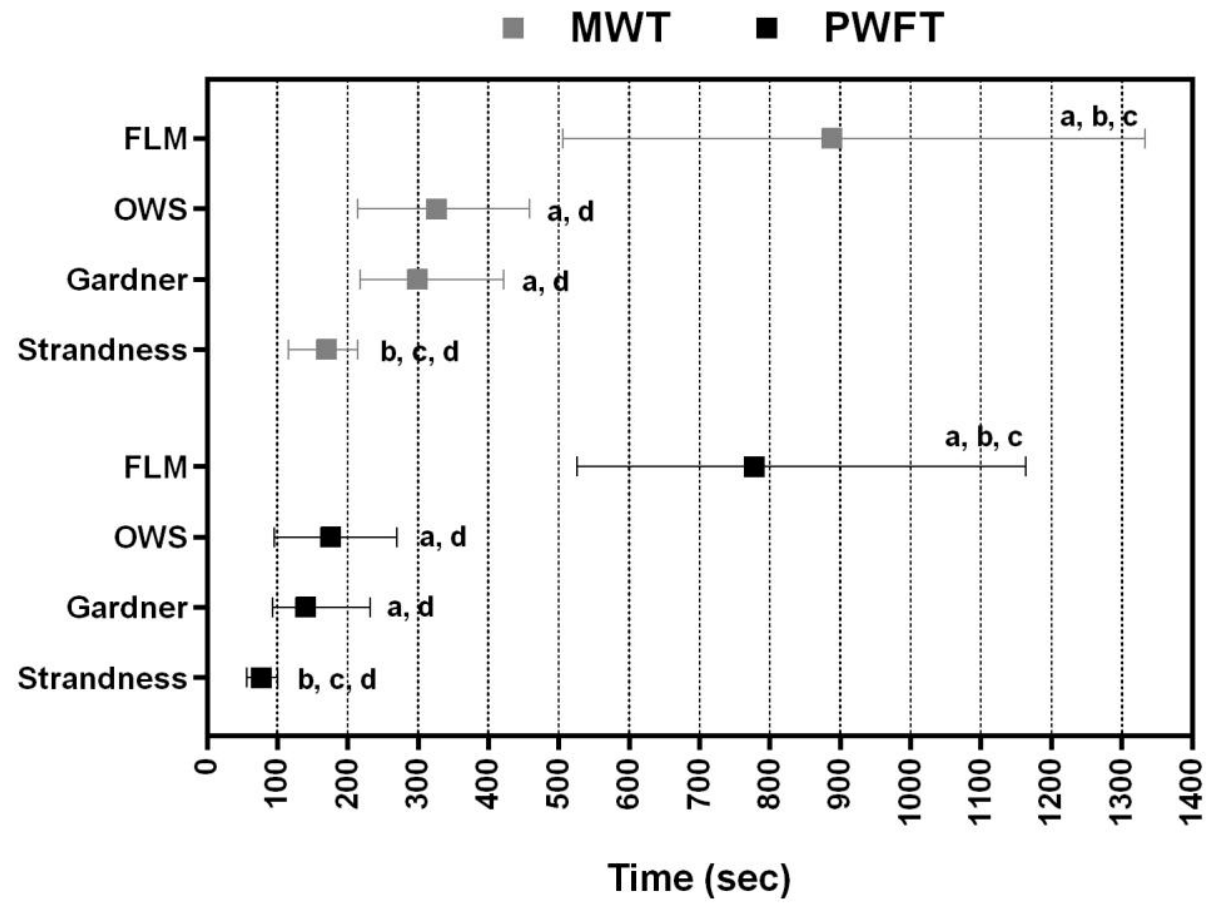


Figure \#3

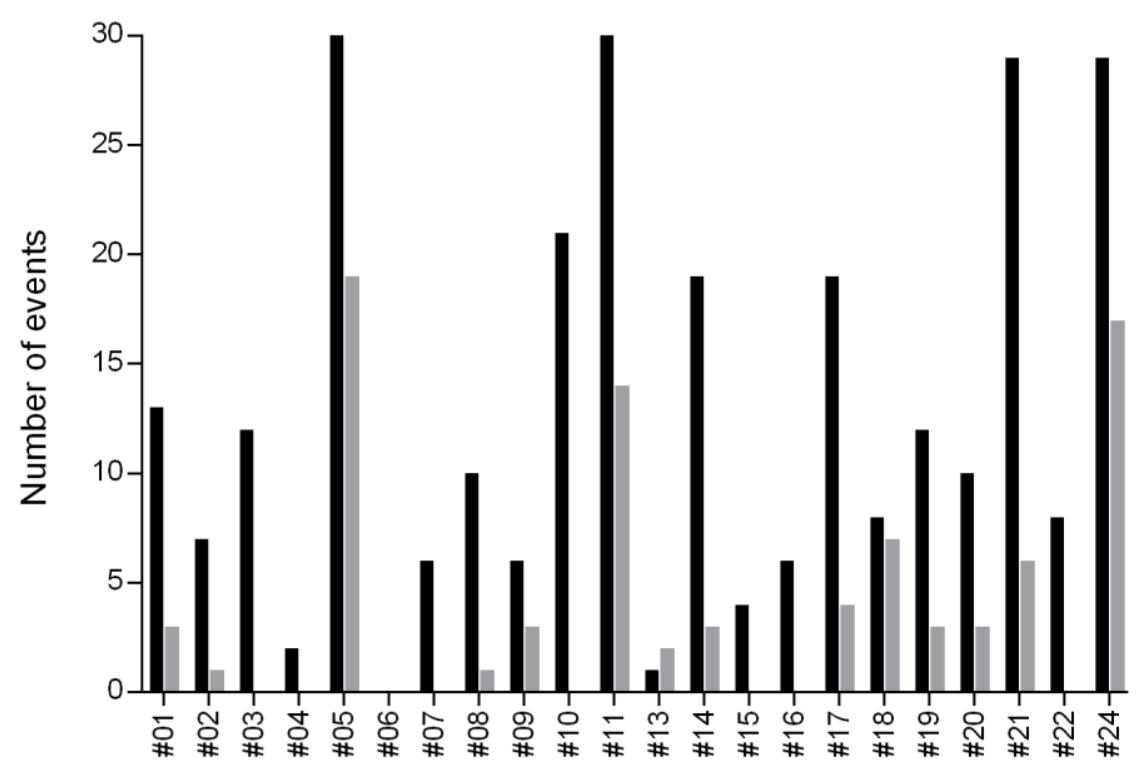

Participant number

- WPM SIMP 